\title{
The crushing of therapies for treating female pelvic floor disorders: the price of physicians failing to lead
}

\author{
Andrew Cassidenti ${ }^{1}$
}

Received: 6 January 2020 / Accepted: 6 January 2020 / Published online: 3 February 2020

(C) The International Urogynecological Association 2020

The FDA's removal of transvaginal mesh from the market last April was enabled by an availability cascade of negative public emotions and press [1, 2], political pressures, plaintiff attorneys and a failure of physician leadership that did not zealously advocate for our patients.

In April 2016, when litigation and negative public media attention led Astora Women's Health (formerly AMS) to leave the incontinence and prolapse device space having had the most widely used and studied midurethral slings, pelvic organ prolapse repair mesh kits and technologies, I wrote an editorial for OB/GYN Management entitled "The crushing of innovation for treating female pelvic floor disorders: a story of lead or be led." I warned that if physicians did not take immediate proactive measures, we risked losing more innovative therapies that help our patients. I outlined in detail work that needed to be done and stated "it would be a tragedy if all of this work does not lead to fully enrolled and completed 522 studies so that we can scientifically make decisions on products before any more treatment options are removed from the market" [3].

On April 16, 2019, the FDA abruptly removed all transvaginal mesh (TVM) products from the market before the 522 studies had been completed and the 3-year data analyzed. They stated and used as a headline this was done "to protect women's health." The FDA up-classified TVM to Class 3 in January 2016 and required TVM manufacturers to submit a premarket approval (PMA) application by July 2018 to support the safety and effectiveness of TVM for POP. Boston Scientific and Coloplast both complied and met the deadline. The FDA moved the goal posts by changing 522 study criteria for TVM from noninferiority to superiority over native tissue after an FDA advisory panel was convened in February 2019 and recommended this change. This panel

Andrew Cassidenti

abruin4ev@aol.com

1 Kern Medical Center, Bakersfield, CA, USA behaved as if their decision to terminate TVM was predetermined, and they limited testimony to that effect. The FDA moved the goal posts even further back when they removed TVM from the market before the 522 studies had been completed, citing federal law timeline mandates. In addition, they insisted that the companies complete the 522 studies but told them if the 522 data show TVM superiority to native tissue, the companies would need to perform a completely new randomized superiority endpoint trial of $>5$ year duration involving a diverse population of clinical trial sites (not just research centers) with blinded POP-Q assessments all performed while the products are still off the market. By doing so, the FDA has completely removed the goal posts from the playing field and has done a great disservice to our patients and skilled vaginal surgeons who were expert in TVM. They put the death nail in this innovative product and have established a frightening precedent that threatens future innovation in products to treat female pelvic floor disorders and discourages industry to invest in this space. An online petition signed by thousands of surgeons protesting the FDA mesh ban before the completed 522 study science could be evaluated was discounted and ignored by the FDA who replied they do not respond to online petitions.

TVM for the anterior compartment and vaginal apex is mesh placed in the vesicovaginal space for anterior wall support with attachment of supporting mesh arms to the sacrospinous ligament. Sacrocolpopexy (SC) is mesh placed transabdominally into the vesicovaginal space and anchored to the anterior longitudinal ligament of the sacrum. To achieve best surgical outcomes, both procedures require placement of mesh in the same vesicovaginal space. With TVM, one remains retro-peritoneal the entire time, which is safer than delivering the mesh transabdominally as with SC. TVM is a more minimally invasive apical and anterior vaginal wall mesh repair requiring only one vaginal incision. Women with previous abdominal surgeries and bowel disease that make abdominal surgery high risk benefitted greatly from TVM delivery as well. 
I believe TVM has received more scrutiny and publicity than SC for two reasons. First, SC has historically been done by few, highly skilled surgeons as the suturing near the sacrum appropriately intimidated the majority of gynecologic surgeons as they feared injury to the middle sacral artery and left common iliac vein, which can be catastrophic and has been fatal. TVM dissection avoids these dangerous vessels and hence more gynecologists felt comfortable doing the surgery. Second, there was a technical evolution and longer learning curve for physicians to perform the necessary full thickness vaginal wall dissection necessary to access the vesicovaginal space transvaginally, which is critical to achieving excellent results with TVM. Early TVM mesh placement was in a split thickness vaginal wall dissection and resulted in the observed higher mesh exposure and vaginal pain rates. I did not start using TVM until I had developed a technique with which I could reproducibly access the vesicovaginal space transvaginally. I shared my technique with a video presentation at the 2011 Society of Gynecologic Surgeons meeting entitled "Accessing the vesicovaginal space transvaginally for optimal POP mesh placement," which remains available on the SGS website video library. Even with this very reproducible technique, not all gynecologic surgeons have the skill set or surgical volume to master it, nor should they try. Surgical outcomes have been shown to be related directly to surgical volumes and experience [4], and TVM should only be done by skilled, high-volume surgeons to optimize results and minimize complications. I believe that has been the case for the past few years, and the early 522 data support this as well.

With TVM kits removed, surgeons may cut their own pieces of mesh from commercially available mesh sheets and place it transvaginally. Surgeons would likely cut shapes similar if not identical to the shapes of the mesh kits they had great success with and then would expose themselves to a more challenging defense if there were to be a complication and litigation. Hence, the only undisputed option now to deliver mesh is transabdominally with $\mathrm{SC}$ whose complications (middle sacral artery bleeding, left common iliac bleeding, death, bowel obstruction, ureteral injury, discitis, apical mesh exposures requiring transabdominal surgery again) are more severe, morbid and difficult to manage than TVM complications. I predict a significant increase in $\mathrm{SC}$ complications as vaginal surgeons learn and master $\mathrm{SC}$, which would have been avoided if TVM had been left in place. The FDA will have to accept some responsibility for these unintended consequences. My fear is a spike in SC complications could start a chain of events that may eventually lead to the FDA banning $\mathrm{SC}$ mesh as well.

I suggest that if the FDA truly wants to "protect women's health," they should honor the criteria of the initially designed 522 studies that they signed off on. I respectively request they allow TVM to be reintroduced into the market if the data warrant it without further expensive and prohibitive studies forced onto industry.

The midurethral sling is at risk as well [5]. For the necessary steps needed to prevent further loss of therapies and innovation, I'll refer you to my 2016 editorial [3] and hope we now decide to lead instead of being led.

\section{Compliance with ethical standards}

Conflict of interest Prior consultant and proctor for AMS and Astora's Women's Heath. Current consultant, proctor and expert witness for Boston Scientific in the mesh litigation.

\section{References}

1. Kuran T, Sunstein C. Availability cascades and risk regulation. Stanford Law Rev. 1999;51:683-768.

2. Rosenbaum L. N-of-1-tradegy, trade-offs, and the demise of morcellation. N Engl J Med. 2016;374(10):986-90.

3. Cassidenti A. The crushing of innovation for treating female pelvic floor disorders: a story of "lead or be led.". OBG Manag. 2016;28(4): 9-14.

4. Meyer CP, Trinh QD. Complications after surgery for stress incontinence: untangling a mesh of uncertainties. JAMA Surg. 2015;150(12):1175-6.

5. Swift $\mathrm{S}$. What if you could not do a mid-urethral sling? Int Urogynecol J. 2019;30:1387-8.

Publisher's note Springer Nature remains neutral with regard to jurisdictional claims in published maps and institutional affiliations. 